\title{
Enhancing the Administration of National Examinations using Mobile Cloud Technologies: A Case of Malawi National Examinations Board
}

\author{
Lovemore Solomon \\ Department of Computer Science \\ University of Zambia \\ Lusaka, Zambia
}

\author{
Jackson Phiri \\ Department of Computer Science \\ University of Zambia \\ Lusaka, Zambia
}

\begin{abstract}
Technological advances and the search for efficiency have catalyzed recently a migration from paper-andpencil based way of doing things to computer-based in education and training at all levels with its drivers being faster administration, processing and delivery of examination results, error free marking of test items and enhanced interactivity. This research paper aims at establishing the challenges currently faced by Malawi National Examinations Board (MANEB) when registering candidates for national examinations as well as disseminating examinations results. A Short Message Service/Unstructured Supplementary Service Data (SMS/USSD) based mobile application using cloud infrastructure is proposed to address the challenges. Data was collected from 80 respondents consisting of teachers, parents and students whose analytical results show that current MANEB business processes have a number of irregularities that subsequently result in candidates' registration records missing or being incorrect as well as delayed access to examinations results by candidates. The proposed SMS/USSD application was tested and proved to be faster and more reliable than the traditional computer based approach that is currently being utilized.
\end{abstract}

Keywords-National examinations; Short Message Service (SMS); Unstructured Supplementary Service Data (USSD); candidate; cloud computing; Malawi National Examinations Board (MANEB)

\section{INTRODUCTION}

In almost every nation, public examinations tend to put forth the enormous influence on the nature of learning and teaching. They dictate not only what is taught but also how it is taught. Educational assessment is understood to be concerned with determination of progress that students have made towards educational goals at a particular time. It is also understood to be an evaluation on the extent to which set out educational goals and objectives are met over a period of time. The main function of these public examinations is to select students for the next highest level of the education system [1]. Globally, many school going children are accessing education as a result of development and implementation of policies aimed at increasing enrolment in schools. While this may be a positive move in as far as development of human capacity is concerned, all these children have to sit for national examinations at the various levels of the education system, resulting in a tremendous increase in candidature. This poses numerous challenges to national bodies that are constituted to organize as well as administer these national examinations [2].

There has been increasing concern in the rising of learner absenteeism from public examinations in many countries across the globe. Research has been conducted to examine the extent, trends and cause of learner absenteeism during public examinations at various grades of primary and secondary education. A number of these studies reveal that the causes of such absenteeism are either system related factors, school related factors or home background factors [3]. There are several system related factors that contribute to the phenomenon of learners failing to sit for national examinations. One of the factors is the process of administration of various national examinations that these learners are supposed to take at various levels of the education system as designed for a particular country. When learners fail to sit national examinations for a particular year, they are likely to drop out of school or repeat that grade.

The Malawi National Examinations Board (MANEB) is a government statutory body that was enacted by act of parliament in 1969 to administer schools national examinations in Malawi. It administers three main national examinations namely Primary School Leaving Certificate (PSLCE), Junior Certificate Examinations (JCE) and Malawi School Certificate of Education (MSCE) [4]. By setting and administering highstakes public examinations that are based on the school curriculum, the Board is capable of providing the needed influence for achieving educational goals [1]. Although the so called e-registration was introduced some years back, MANEB still experiences a number of challenges when administering these national examinations. This largely is because of the pressure that MANEB faces to accommodate the ever increasing candidature of national examinations due to the introduction of free primary education. Apart from basic or lower grades (i.e. standards 1, 2 and 3), high levels of school dropout as well as grade repetition in Malawi are also observed in grades where students sit for national examinations [5]. Notwithstanding the fact that a lot of students fail in these examinations and that others fall short of writing because of several other reasons, it is highly evident that the way these examinations are administered contributes to the number of students who fail to sit these examinations. 
The administration of these examinations is associated with a big challenge in making sure that all the students who register for the examinations in a particular year have their registration details properly captured and that they are not missed out. The current system of registering students uses Microsoft excel spreadsheets and CDs/DVDs to gather and transfer data from one point to another within the registration process. This leads to data loss as well as data incorrectness as the tools used are not reliable. Secondly, when students register with their respective schools or centers, there is no proper and straightforward means of letting registered students verify, and in case of anomalies, rectify their registration details before the date of examinations. As a result of these shortfalls in the current process, each year a good number of candidates fail to sit national examinations because their registration details are either incorrect or are completely missing. The incorrect details include names, age, sex, identification numbers, school numbers as well as subjects registered and paid for. When this happens, students have no option but to wait and re-register the following year thereby repeating the class. If they do not have money to re-register, they just dropout. MANEB also faces another big challenge when it comes to dissemination of examinations results to students when marking and grading is completed. Apparently, MANEB produces nominal rolls and/or hard copy books that are sent to schools as well as District Education Offices where candidates check their results from. Apart from wastage of resources in the form of stationery, this method of results dissemination usually takes long to be accomplished and as a result examinations results are always delayed. These problems, that have been there since MANEB's inception motivated the researcher to carry out the study to establish the challenges and their real cause and subsequently propose a solution to the challenges using modern technologies.

Administration of national examinations is a collection of processes that include registration of students for examinations, verification of registered students' details before examinations, preparation of examinations, assessing and grading students as well as release and dissemination of examination results. This study only focuses on the registration and verification of students as well as dissemination of examinations results. The study proposes an SMS/USSD mobile application that uses the Global System for Mobile communication (GSM) infrastructure provided by local mobile service providers. The SMS/USSD approach is preferred as it is easy to use considering the diverse literacy levels of the intended users that include people in villages who can only afford to write messages and make phone calls with a mobile phone. Secondly, SMS/USSD can be used with any mobile phone be it feature or smart phone. The proposed system also has a web interface for the system administrator and other users for data management and manipulation. It is deployed in the cloud for data safety and reduction of costs to be incurred by MANEB to have the system implemented.

The remainder of the paper is structured as follows. Firstly, theory related to the study is discussed in Section II. The theory is grouped into literature reviewed and related works which are provided in subsections II(A) and II(B), respectively. Section III looks at the methodologies and tools that were used to carry out the research and design the proposed system. It covers the baseline study, system requirements and business process modelling. Next, the paper outlines the results of the baseline study as well as the system prototype development in section IV. Section V discusses the results observed in the previous section in subsection $\mathrm{V}(\mathrm{A})$ and provides a conclusion in $\mathrm{V}(\mathrm{B})$. Here the researcher gives a general interpretation of the results with regard to the objective of the study. Section VI looks at the researcher's recommendations and suggested future works while Section VII is the acknowledgement.

\section{THEORY}

\section{A. Literature Review}

Effective policies to improve school progression and reduce the numbers of children dropping out of school are critical if Universal Primary Education (UPE) is to be achieved. School dropout has been defined as leaving education (in this case primary and secondary school) without obtaining a minimal credential (most often a higher secondary education certificate) [6]. On the other hand, grade retention/repetition is the practice of holding a student/learner in the same school grade or class for an additional year rather than promoting them to the next level grade with their age peers [7]. Research on the causes of school dropout and grade repetition has been done all over the world. Results indicate that school dropout as well as grade retention is an issue that can be an indication of the academic, intellectual and social economic level of the students but also as the success level of the education system [3]. Generally, national education plans assume that school progression will improve automatically as a result of interventions designed to improve initial access to educational quality, which is not the case. There is not one single cause of dropout rather it is often a process and therefore has more than one proximate cause. Apart from the most general causes of dropout and repetition, the system of educational provision generates conditions that can ultimately input on the likelihood of children to drop out from school [8].

Properly managed national examinations can significantly reduce the number of dropouts and repeating students in grades that sit for national examinations. Presently, the examination system is one of the key contemporary moral issues as technology continues to play a transforming role in societies in all over the world. The prospects for the utilization of new technologies in the field of education continue to be part of human consciousness from a number of angles one of which is that of public examinations. ICT is exerting a powerful influence on almost every facet of life. However, insufficient attention has been paid to the relationship between formal assessment and use of ICT (Harding and Craven, 2001). Accordingly, the education system can be the most effective sector to anticipate and possibly eliminate the negative impact of ICT [9].

ICTs have become within a very short time, one of the basic building blocks of modern society. Many countries now regard understanding ICT and mastering the basic skills and concepts of ICT as part of the core of education, alongside reading, writing and numeracy [10]. According to (UNESCO, 2002), Information and Communications Technology may be regarded as the combination of informatics technology with 
other related technology, specifically communication technology. Informatics refers to the science dealing with the design, realization, evaluation, use and maintenance of information processing systems, including hardware, software, organizational and human aspects and the industrial, commercial, government and political implications of these. Hence Informatics Technology can be defined as the technological applications (artifacts) of information technology [10].

The National Institute of Standards and Technology (NIST) defines cloud computing as a model for enabling convenient, on demand network access to shared pool of configurable computing resources i.e. networks, server, applications and services that can be rapidly provisioned and released with minimal management effort or service provider interaction [11]. Cloud computing has become one of the most significantly achieved developments in the IT industry which gives a platform to use applications in the form of services which is more scalable, reliable, high performance and relatively low cost as compared to the other distributed computing infrastructure. It is increasingly accepted that in the future most information sources and desktop applications currently in use will be mainly accessed through the internet, now increasingly referred to as 'the cloud'. The web software and cloud computing will definitely have an impact on enterprise IT, but the impact on the educational system will be astounding and many in educational system don't see it coming. These trends are moving much faster than the current educational systems can handle [12]. Uniquely from other distributed system paradigms, cloud users are not required to have knowledge of, expertise in or control over the technology infrastructure that supports them [13]. A cloud computing system has got the front end and the backend sections. The front end is where the computer user or client sees. It includes the computer and the applications required to access the cloud computing system. The backend is the cloud section of the system where there are various computers, servers and data storage systems that create the cloud of computing services. A central server administers the system, monitoring traffic and client demands to ensure everything runs properly. The system follows a set of rules called protocols and uses a special kind of software called middleware that allows networked devices to communicate with each other. The applications, services, data and infrastructure in the cloud have certain features that define them i.e. services and data are remotely hosted on remote infrastructure, they are ubiquitous meaning that they are available anywhere and also the pay-per-use feature that brings in utility computing model similar to that of traditional utilities and electricity where one pays for what they use [14].

Globalization makes governments establish e-government, a hype that has attracted many businesses, organizations and institutions that require collaborative, flexible, scalable and cost effective computational infrastructure [15]. With the explosion of mobile applications and the support of cloud computing for a variety of services for mobile users, mobile cloud computing (MCC) is introduced as an integration of cloud computing into the mobile environment. Mobile cloud computing brings new types of services and facilities for mobile users to take full advantages of cloud computing [16].
Mobile cloud computing is an integration of cloud computing technology with mobile devices to make the mobile devices resource-full in terms of computational power, memory, storage, energy and context awareness [17]. In the past, all services on the internet were available only to computer users. Mobile phones are greatly expanding the market for the electronic delivery of services available on the internet [18]. Mobile data communication is already extending the internet to people on the move. Facilitating this will be a new technology called Wireless Application Protocol (WAP), which will, to put it simply, bring the internet to a mobile phone. The latest web computing-related technologies (including WAP, WML, Script and CGI programming) are gaining interest for applications in software technology research and education. Implementing a practical internet application system to effectively utilize and evaluate these technologies can actually be combined for substantial improvement of productivity to end users as well as providers [18].

Short Message Service (SMS) technology evolved out of the Global System for Mobile Communication (GSM) standard, an internationally accepted cell phone network specification the European Telecommunications Standards Institute created. The dramatic penetration of mobile phones all over the world, even in very remote areas, has created enormous opportunities for marketers and public administrators to be in touch with consumers with continuous interactivity so that citizens can find a satisfactory relationship to government [19]. Text messages are short alphanumeric communications sent from one mobile phone user to another with messaging applications on mobile handsets. They are frequently used for social coordination and personal communication because text messages are quick and cheap to send [20]. SMS gateway can be defined as a system or mechanism that facilitates SMS transition by transforming the messages from several types of communication media to mobile network traffic, in vice versa, allowing, receiving and transmitting the SMS messages with or without the use of a mobile phone. The typical working process of SMS gateway system is similar to the concept of regular email or SMS in terms of a system receives a message from sender client then conveys it to the receiver client [21]. SMS messages are handled via a Short Message Service Center (SMSC) that the cellular provider maintains for the end device. SMSCs operate in either a store - and - forward or a forward - and - forget paradigm. In the former paradigm, the system resends the message for some period of time until it is successfully received. In the latter paradigm, the system sends the message to the end device without assurance of receipt or an attempt to redeliver in the case of failure [22]. SMS applications can either be independent or dependent services. Independent service involves using solely a mobile phone and the application server (system running SMS application). The mobile phone uses a regular SIM card which has a normal phone number and messages that originate through the phone attract the standard cost or tariff. On the other hand, Dependent service involves having the application server connect to the service provider's SMS center (SMSC). It requires a constant connection to the internet as the application server does not require any physical phone/modem with a SIM card connected to it; rather it connects to a SMSC. When users send their 
requests, they go to the SMSC which automatically forwards the message to the application server over the internet. This option provides added benefits as the service provider can provide a special tariff and a dedicated line for the application [23].

SMS communication happens between Mobile Equipment (ME) and the Application Server (AS). The Application Server deals with the content of SMS from the ME, and the SMSGateway is a gate way which receives the SMS from a ME or sends the SMS to a ME by the connection with SMSC. AS and SMS-Gateway as well as resource database are located in local network environment of the Service Provider (SP) [24]. When a handset sends out an SMS (possibly a requesting message), it is firstly delivered from the handset (ME) to a SMSC through the base station (BSS). Then the SMSC gets the destination of the SMS and forwards the message to AS through the SMSGateway or SMS-Gateway Mobile switching Center (SMSGMSC). The SMS-GMSC will then access the Home Location Register (HLR), search to locate the cellular phone address at the end point, and then route information to the Mobile Switching Center (MSC). After receiving the data, the MSC will determine which SMSC to contact for this end point. If the caller is on roaming mode, SMS-Internetworking Mobile Switching Center (SMS-IWMSC) will be the message's next stop [25]. On the contrary, when a SMS message (possibly a response message) is sent to a mobile user by AS, it is firstly delivered from AS to the SMSC through the SMS-Gateway; the SMSC then broadcasts the message through the BSS to the destination ME. The connection between SMSC and the BSS is over the SS7 network and that between SMSC and the SMSGateway is facilitated by a TCP/IP connection over the Internet [24].

Unstructured Supplementary Service Data (USSD) applications are accessed by user request, and make use of short codes or text strings to trigger certain services in a session-based communication. These codes could perform a function, request a snippet of information, or lead the user into a series of textual menus which are navigated through the corresponding menu numbers. Knowingly or unknowingly, USSD is used by virtually every mobile owner by entering a code starting with asterisk (*) and hash (\#) on a mobile phone such as for the purpose of checking balance in prepaid cell card or weather forecast [30]. While SMS is based on the characteristics of storing and forwarding data, USSD is session based and real time. [29] outlines some of the reasons why USSD is preferred over other existing technologies as follows:

- Session-oriented, unlike SMS, which is a store and forward, transaction oriented technology.

- Turn-around response times for interactive applications are shorter for USSD than SMS because of the session based feature of USSD.

- Users do not need to access any particular phone menu to access services with USSD. They can enter USSD commands direct from the initial mobile phone screen.

- USSD commands are routed back to the home mobile network's Home Location Register (HLR), allowing for the virtual home environment concept to work just as well and in exactly the same way when users are roaming.

- USSD works on all existing GSM mobile phones.

- Both SIM Application Toolkit and the Wireless Application Protocol support USSD.

\section{B. Related Works}

Apparently, there are quite a number of solutions to SMS/USSD based data acquisition and dissemination systems. Therefore, the prototype model utilizes similar technologies where a mobile phone communicates with an application residing on a server within the cloud through a mobile service provider.

Boukas [26] presented a fully-functional SMS-oriented mobile informational system called Pandora. The system was designed and deployed from the onset to specifically support a plethora of services obtainable mainly by the students of university of the Aegeen in Greece. It offers administrative, informative and strictly academic services. Administrative services include subscribing and unsubscribing users etc. and informative services include news, weather broadcast etc. while strictly academic services include registration for courses, boarding, phonebook, etc.

In the literature, Shamsuzzaman et al. [27] studied a system to improve the vaccination coverage in hard-to-reach areas of Bangladesh. It consisted of a web database application named 'mTika' that works with Android smartphone application used to register pregnant women. The web database has a module for SMS vaccination reminders to mothers.

Prajakta [28] on the other hand proposed an online healthcare system using cloud computing. It is an integrated system to have one's health checkups done quickly and access reports anywhere anytime on the phone and patients can contact specialized doctors in their locality from the phone. Even if a patient requires blood, he/she makes the request to the system using the phone and the application broadcasts the request to people with same blood group in the locality.

Awodele [23] proposed an SMS based system to provide a means of cheap and fast communication between the students and the university. It works using client-server architecture and deployed as a dependent service i.e. the server (with SMS application) has a phone with a standard SIM card connected to it. The SMS server receives SMS messages from users and processes the message by connecting to the database that holds the details and grades.

Another solution was proposed by C.L. Tseng to solve the problem of manual practice of collecting and managing farm field data by humans [25]. The solution uses GSM and SMS to conduct field data acquisition. It includes an automatic field data collecting subsystem called the field monitoring platform (FMP) and a remote host control platform (HCP). The FMP consists of electronic short sensing modules, GPS module, GSM module, environmental parameters sensing module and integration kernel module. The integration kernel module of FMP uses universal synchronous/asynchronous receivertransmitter (USART) to connect with all environmental sensing modules and perform data assembly, processing and 
sequencing functions on the field data received. The GSM module assists with the sending of data wirelessly across the field. The HCP, upon receiving the data that carry all the field information, it decodes the data and save them into the database for future long-term monitoring and statistical analysis to provide a reference framework for future farming improvement.

In China, a medical information query system based on USSD was suggested by $\mathrm{Z}$. Wang and $\mathrm{H}$. Gu to be accessed by mobile phone users within China mobile GSM network which covers $85 \%$ of all Chinese mobile phone users [31]. The system is mainly composed of a USSD service application server, a database and a USSD platform. The USSD service application server realizes USSD service with the information provided by the USSD platform, including information query and fee charge. The medical information data of users is stored in the database. A signal processing platform included in the USSD platform connects to the mobile communication network vial the No. 7 signal channel. The signal processing platform connects to a server-processing platform by highspeed LAN bus to access the USSD service. The USSD service application server connects to the USSD platform in a client/server mode.

\section{MATEREALS AND METHODS}

\section{A. Baseline Study}

The baseline study was conducted to ascertain the challenges faced by MANEB, schools as well as students as regards the registration for national examinations and also disseminating/accessing the examinations results.

1) Study Population: The target population for the study included students who had previously sat any of the national examinations, teachers who are responsible for registering students for national examnations in schools as well as parents with children who had previously sat national examinations.

2) Sample Size and Sampling procedure: The study was done in two of the four administrative regions in Malawi. The regions were randomy selected. Again, five districts from each region were also randomly selected, i.e. ten districts were considered for the study. Lilongwe, Dowa, Salima, Mchinji and Dedza were selected from the central region while Blantyre, Chiradzulu, Mwanza, Neno and Chikwawa were selected in the Southern region as shown in Fig. 1 and 2. Two schools from each district were purposively selected so that one was located in urban while the other in rural areas. Students as well as teachers in these schools were also purposively seleted to participate in the study. This was so because the researcher's focus was on particular characteristics of respondents that were of interest to enable the researcher answer the research questions, i.e. those students that had sat for any of the national examinations and also teachers that are responsible for handling national examinations affairs within a school. Parents were also purposively chosen from the ten districts that were considered in the study. The researcher focused on those parents with at least one child who had previously sat for the national examinations. Literacy level and age of parent respondents were also considered. In total, the study had a sample of 80 respondents including 40 students, 20 teachers and 20 parents.

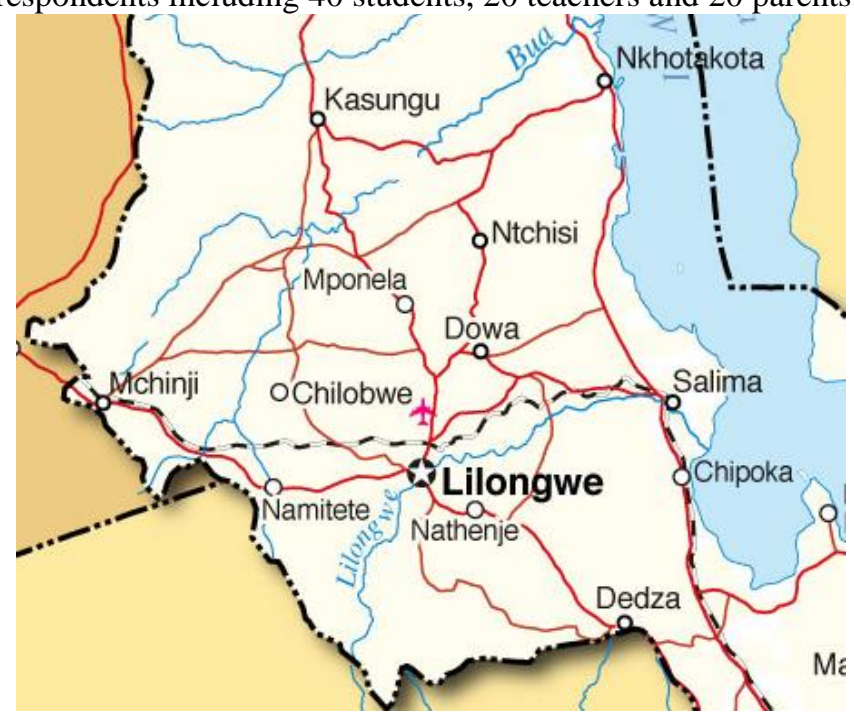

Fig. 1. Districts in Malawi's central region [5].

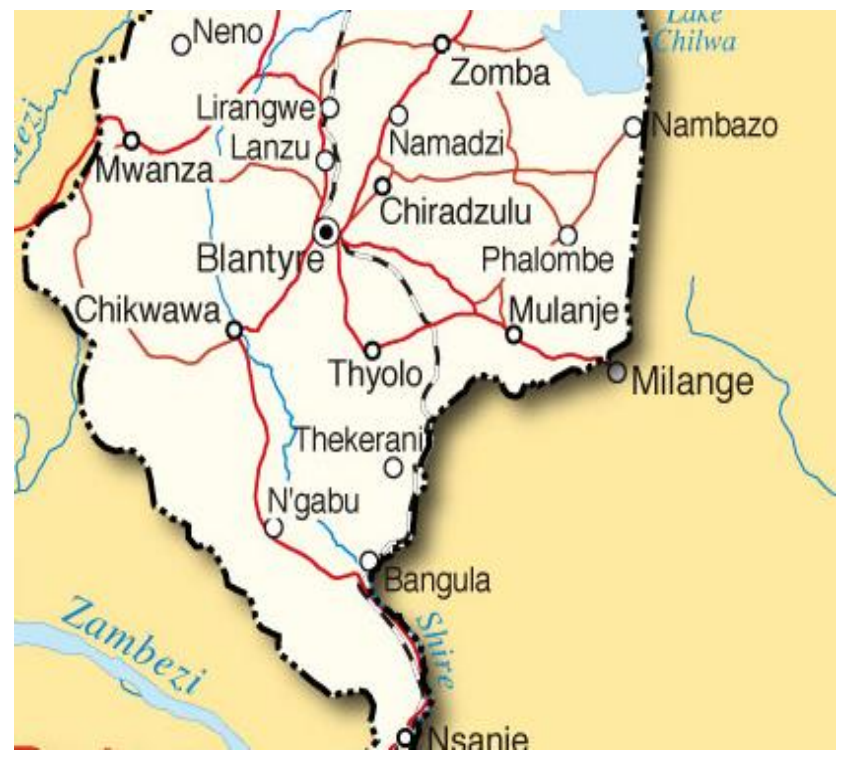

Fig. 2. Districts in Malawi's southern region [5].

3) Research Instruments: The research instruments were designed in a way that they should meet the objective of the baseline study. The instruments included:

- Questionnaire for students who had previously sat for national examinations.

- Questionnaire for teachers who register students for national examinations in schools.

- Questionnaire for parents with children who had previously sat for national examination.

- Interview guide for MANEB and Ministry of Education, Science and Technology (MoEST) employees. 
4) Data Collection: Authority was sought by the researcher from MoEST to collect quantitative data from the sampled schools. Questionnaires were distributed to all the respondents. Respondents were given enough time to respond to the questions. Interviews were also conducted with the relevant participants to gather qualitative data. The data collection exercise lasted for four weeks.

5) Data Analysis: The quantitative data that was collected through the questionnaires was analysed using using computer based software called PSPP, a free program for statistical analysis of sampled data which comes as an alternative to the proprietary SPSS. The outcome and computations were presented in the form of tables, charts and graphs.

\section{B. System Design}

The interviews that were conducted with MANEB and MoEST employees helped to understand how registration of candidates and dissemination of examinations results are currently done. The interviews also helped to gather the system requirements and come up with a model design for the proposed system.

1) Current Business Process: The baseline study revealed that the current process of registering candidates as well as disseminating examinations results by MANEB makes extensive use of paper. Fig. 3 shows the overview of the current business process. Data is physically transmitted from one point to the other using CDs or DVDs. Candidates are registered using excell spreadsheets which are later copied to a CD/DVD or memory stick and delivered to MANEB offices. At MANEB, files from all the schoools across the country are merged into one excle file. Verification of candidates' registration details as well as dissemination of resuts involves printing nominal rolls and hard copy books that are distributed to various schools and offices in all the districts for candidates to check.

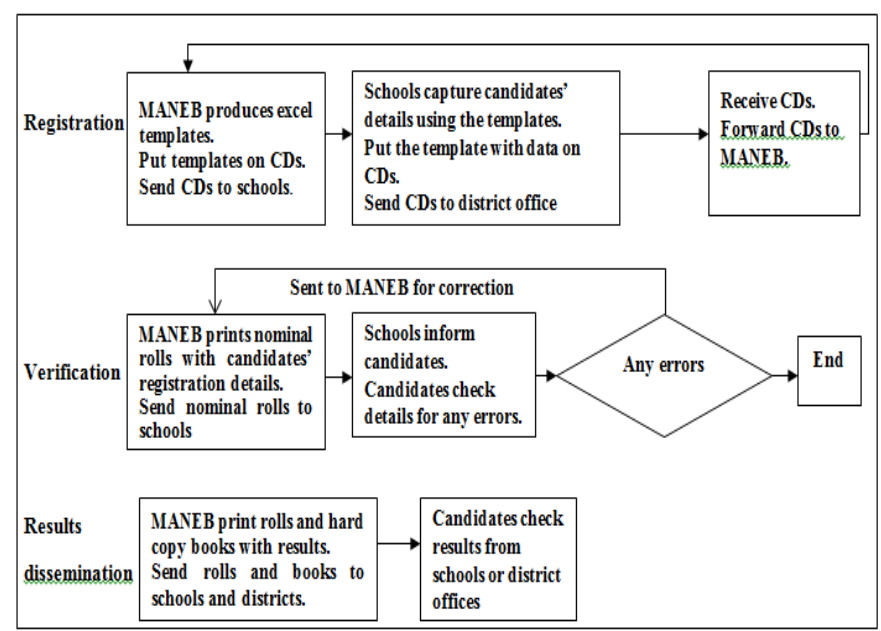

Fig. 3. Current business process.

2) Proposed System Model: The proposed system model utilises the cloud infrastructure and mobile application to register candidates for national examinations as well as accessig examinations results. The system also has a web interface for data manipulation. From the baseline study, it showed that the current system is time consuming and too involving hence the proposed model will help reduce costs and time it takes to register candidates and also access results. The registration data as well as examinations results will be centrally kept in the cloud and accessed when needed by all the stakeholders, i.e. MANEB, MoEST, etc. Students or their parents will be able to register for examinations by simply sending an SMS or invoking a USSD operation with the system. Similarly, examinations results will also be accessed by simply reading an SMS or retrieving them via a USSD transaction. Fig. 4 below depicts the proposed system model.

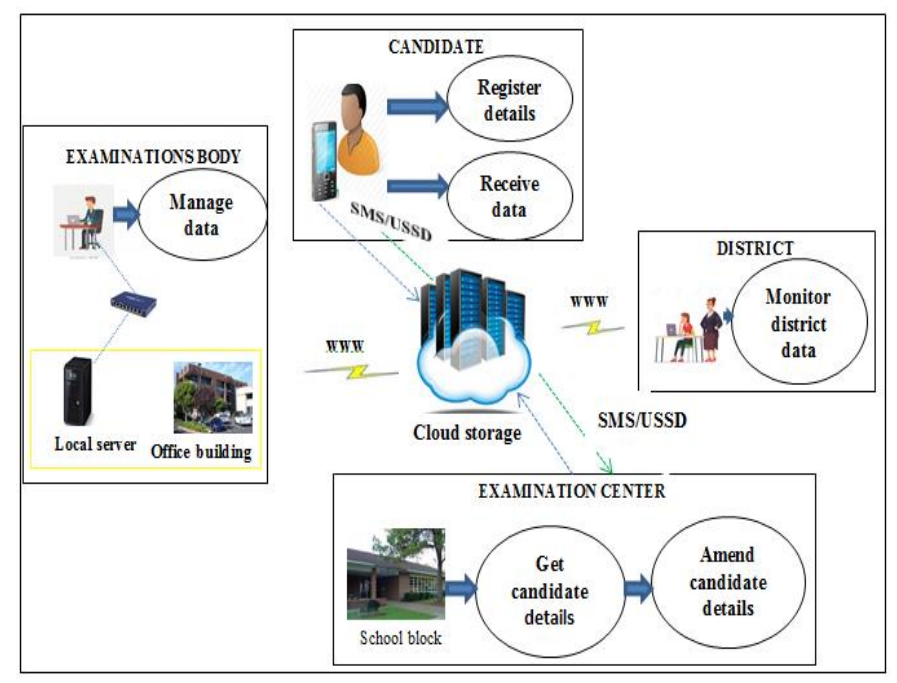

Fig. 4. Proposed registration and results dissemination system.

3) Proposed System Business Process: The study proposed a change by automating the current business process being used by MANEB which is mostly paper based. The system will enable candidates to register for examinations by providing their details i.e. examination number, name, National Registration Card (NRC) number, age, sex, center, district as well as subjects. When registration period ends, the system will push SMSs to all registerd candidates' mobile phones for verification of their registration details. For any anomalies, concerned candidates will have to consult the responsible teacher at their school to amend their details using the USSD functionality of the system. The error correction actitivity will happen within a specified period of time so that when it elapses the system allows no more changes. In a similar way, when marking and grading of candidates' scripts is completed, the results will be imported into the system from where they will be sent to candidates' phones in the form of SMS. Both the registration details and examinations results can also be retrived from the cloud using the USSD facility. When the error correction window closes, the system generates registration cards with barcodes that will be used to validate candidates on the day of writing examinations by scanning them using a barcode scanner. 


\section{System Prototype Development}

The proposed system has two components; SMS/USSD and Web components. A SMS or USSD application requires a gateway which allows a computer to send or receive transactions to and from a telecommunications network. Usually, an individual or organization has to subscribe with a mobile service provider in order to be able to use their gateway, a thing that proved to be expensive to the researcher. As a result, a USSD simulator was developed that sends requests to the database in order to add candidates' registration records and retrieve both registration details and examinations results. The USSD simulator was developed using JAVA programming language. Hypertext Preprocessor (PHP) functions were written to sit between the simulator and the database. These functions actually do accomplish the USSD functionality of the simulator to insert and retrieve data. The Web component of the system prototype was developed using Hypertext Markup Language (HTML) and PHP. The Web application runs on Apache Web server. The system prototype uses MYSQL database engine to store data.

\section{RESULTS}

The results got from the baseline study as well as development and testing of the system prototype are presented in this section. The baseline study was mainly to establish the challenges that MANEB faces when registering candidates for national examinations and dissemination of examinations results. The causes to the challenges were also studied. The proposed system prototype was developed to show the concept of how the fully implemented system would work to mitigate the challenges currently experienced by MANEB.

1) Baseline Study: Data collected was analysed using descriptive statistcs and the results were presented using tables, charts and graphs. The sampled students were asked to rate the current process of registration for national examinations. The results showed that $7.5 \%$ said it is very poor, $55 \%$ said it is poor while $32.5 \%$ said it is good. This is shown in Fig. 5 below.

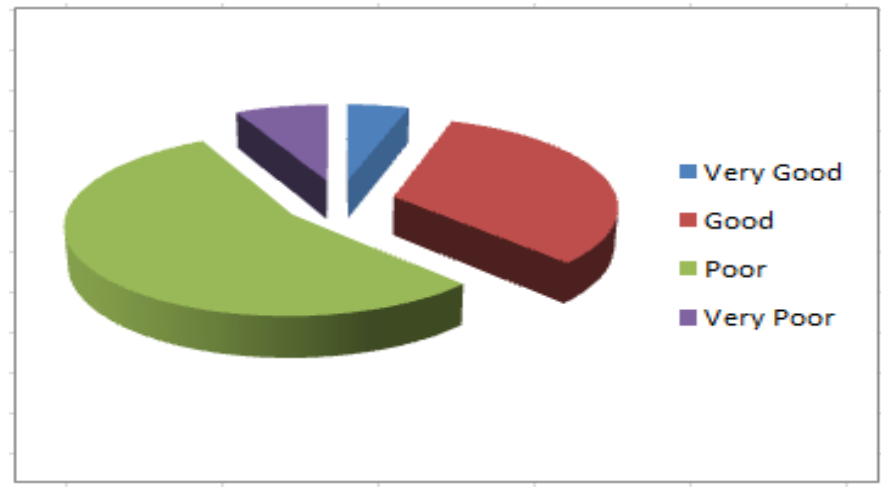

Fig. 5. Students' rating of the current system

Regarding the reliability of verification of candidates' details before sitting the national examinations, teachers in the various schools gave their views as shown in Fig. 6. 55\% said it is not reliable, another $30 \%$ of the teachers said it is somewhat reliable, $10 \%$ said it is reliable while $5 \%$ said it is very reliable.

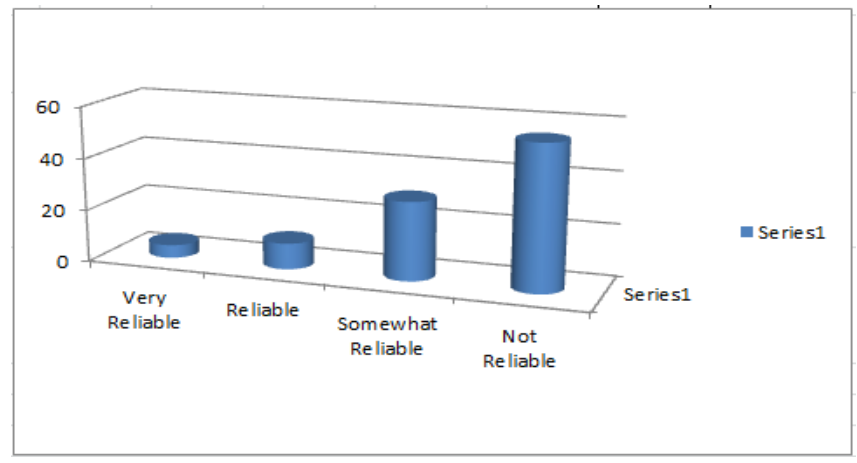

Fig. 6. Reliability of verification process.

Parents were also requested to indicate as to how long it takes them to know their children's' examinations results from the time they are released. Their responses were as shown in Fig. $7.25 \%$ of the respondents indicated that they accessed the results within a day, $55 \%$ said they got the results after some days, $10 \%$ got their results after some weeks and another $10 \%$ of the respondents said results were known after some months.

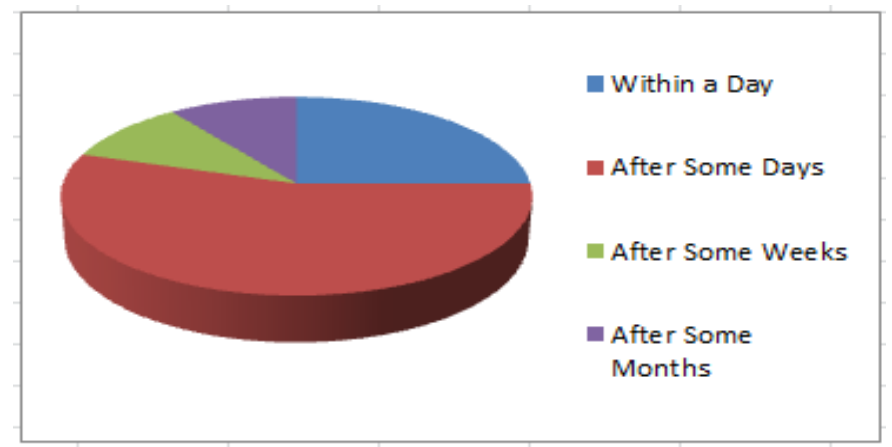

Fig. 7. Time to access results.

Fig. 8 illustrates some of the challenges faced by those teachers who register candidates for national examinations.

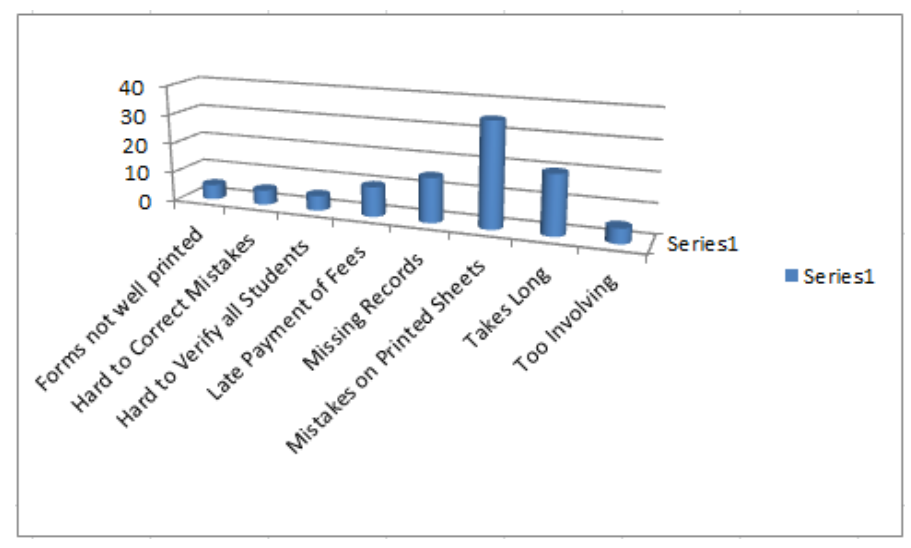

Fig. 8. Challenges with the current process.

The study also revealed that over the years some candidates in some schools have failed to sit their examinations because of the irregularities in the current system. The chart (Fig. 9) below shows the results got from teachers. 


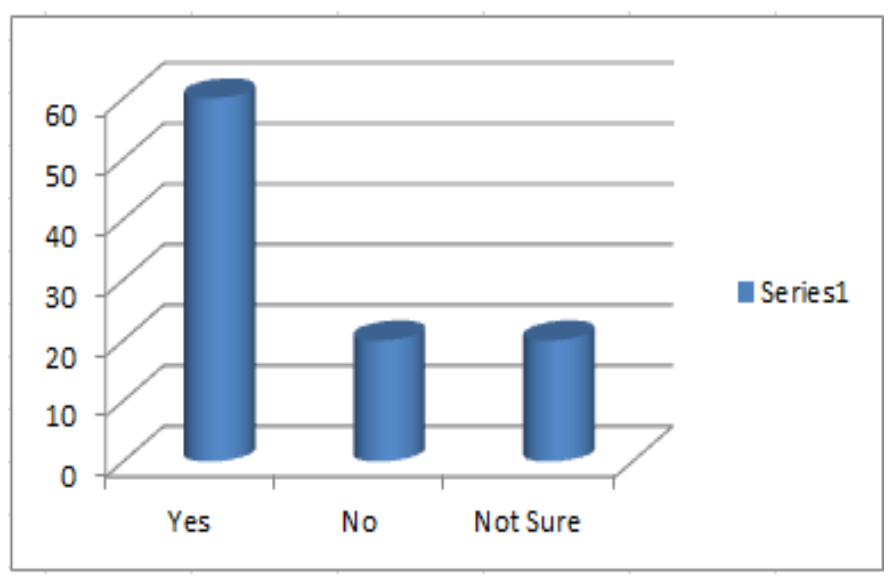

Fig. 9. Failure to sit examinations.

On whether they thought introducing the proposed system would improve the current system, parents responded as indicated in Fig. 10 where $85 \%$ said yes, $5 \%$ said no while $10 \%$ were not sure.

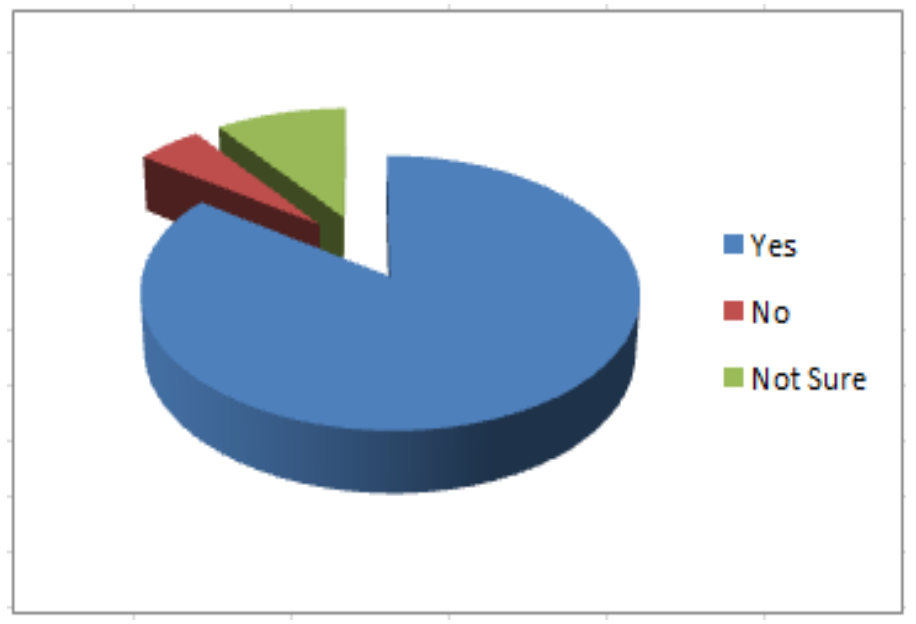

Fig. 10. Parents' perception of the proposed system.

System Prototype Development: As pointed out in the previous section, the system prototype, called Students Data Management System (SDMS) has the SMS/USSD and Web componets. A USSD simulator was develoed using JAVA programming language. The simulator calls PHP functions that perform the USSD tasks by reading the users' choices from the simulator and provide them with the functionality they require according to their choice. In the system prototype, the candidates register their details using the simulator hence the registration is through USSD. A candidate firstly dials a mobile number that is assigned to initiate the USSD session as illustrated in Fig. 11.

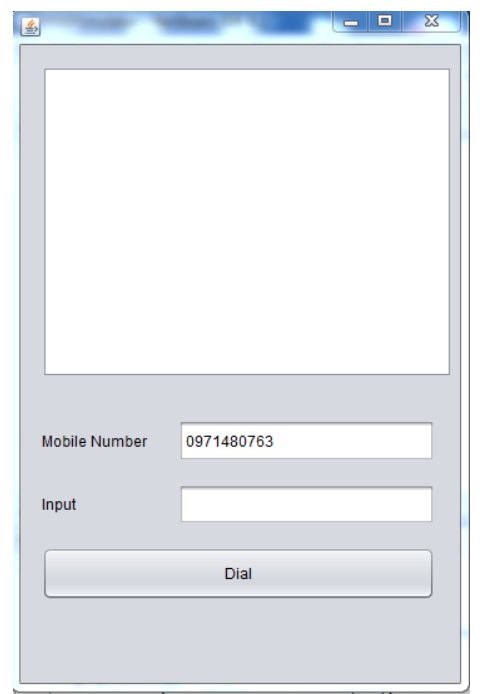

Fig. 11. Initiating USSD session.

Fig. 12 to 15 depicts some of the USSD operations undertaken to register for examinations. After dialing, candidates are presented with a screen displaying the various tasks they want to do. A candidate is expected to input a number against the task to be performed.

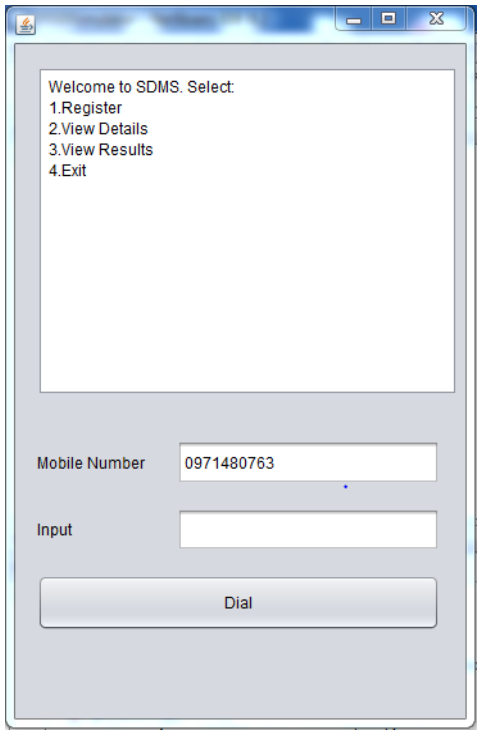

Fig. 12. Screen showing tasks.

When a candidate selects register from the above window, i.e. inputs 1 , he/she is asked to provide the registration details in sequence. Firstly, the candidate chooses the examination type, i.e. PSLCE, JCE and MSCE then proceed providing other details like examination number, names, age, sex, center, subjects, etc. 


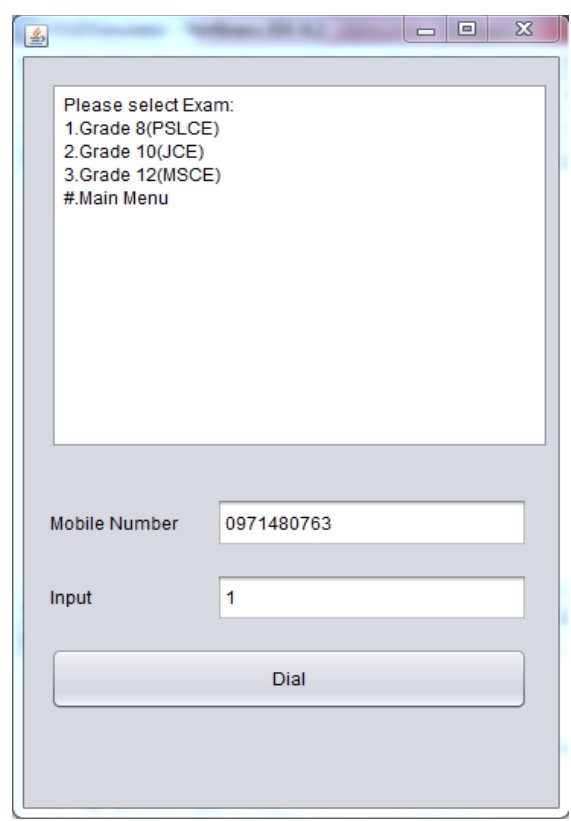

Fig. 13. Screen for examination types.

Examinations number is supplied first followed by all the other required details.

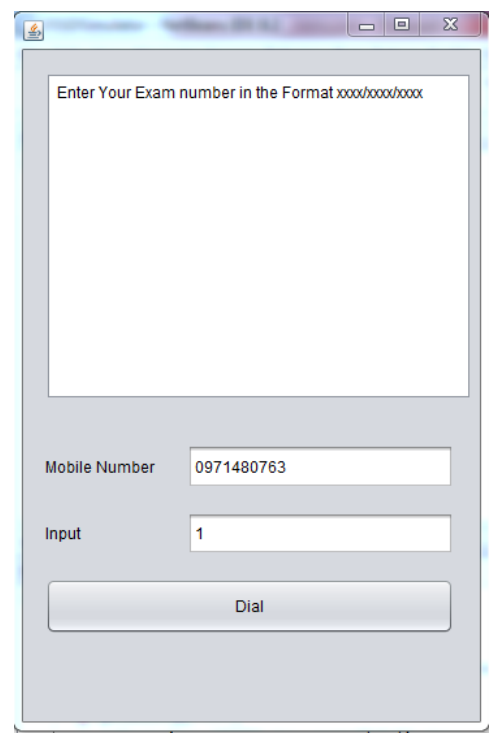

Fig. 14. Screen to enter examination number.

When a candidate supplies all the required details for registration, the system will print a registration successful message.

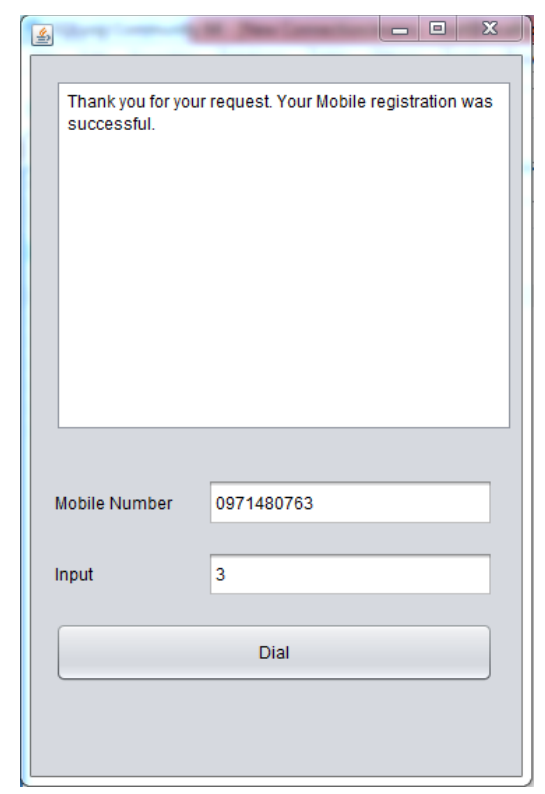

Fig. 15. Successful registration message.

As shown in Fig. 16 below, a candidate is also able to view registration details. To view registration details, a candidate inputs 2 from the initial screen. The candidate will then be asked to provide examination number and the details will be retrieved.

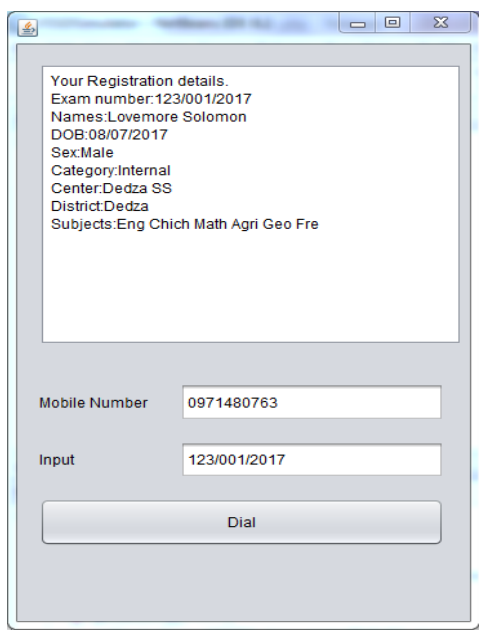

Fig. 16. Screen showing registration details.

Similarly, to check examinations results, candidate inputs 3 from the initial screen and provide examination number to retrieve the results. The check examinations results screen is shown in Fig. 17. 


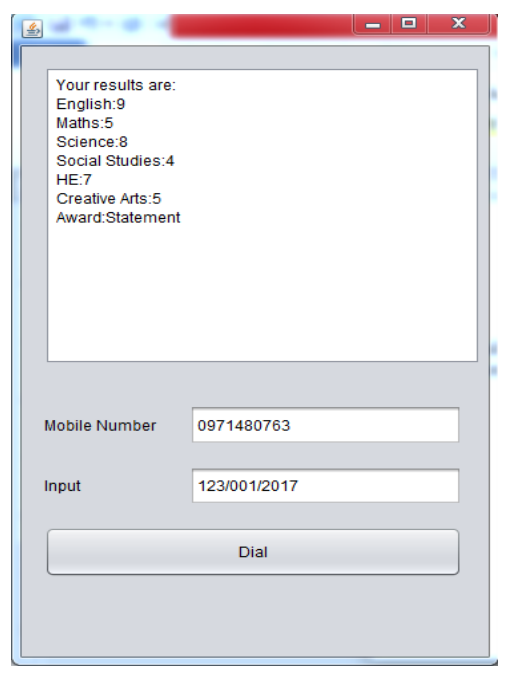

Fig. 17. Screen showing examinations results.

The Web component of the system prototype is accessed through the internet by logging in the system using the log in credentials given to a user. The user logs into the system using the login window (Fig. 18.)

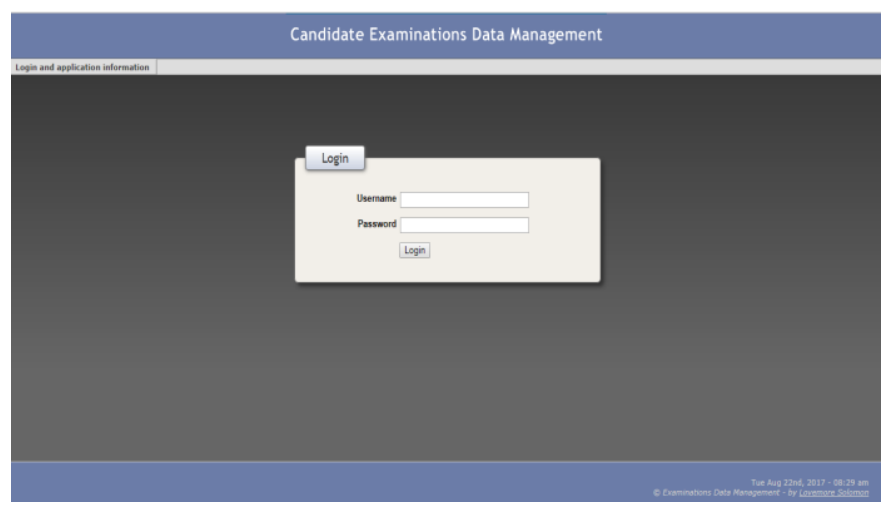

Fig. 18. Web component log in screen.

The system administrator will be responsible for all the administrative activities of the system. Administrator will be able to add, view, update and archive users of the system, taking data backups, adding centers, districts, examinations etc. Fig. 19 and 20 demonstrate adding a user to the system and viewing users of the system, respectively.

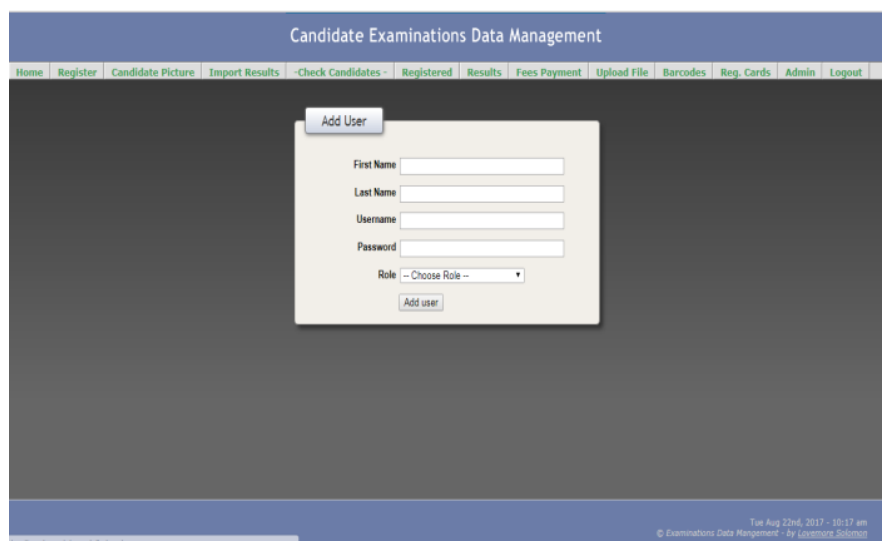

Fig. 19. Adding a user to the system.

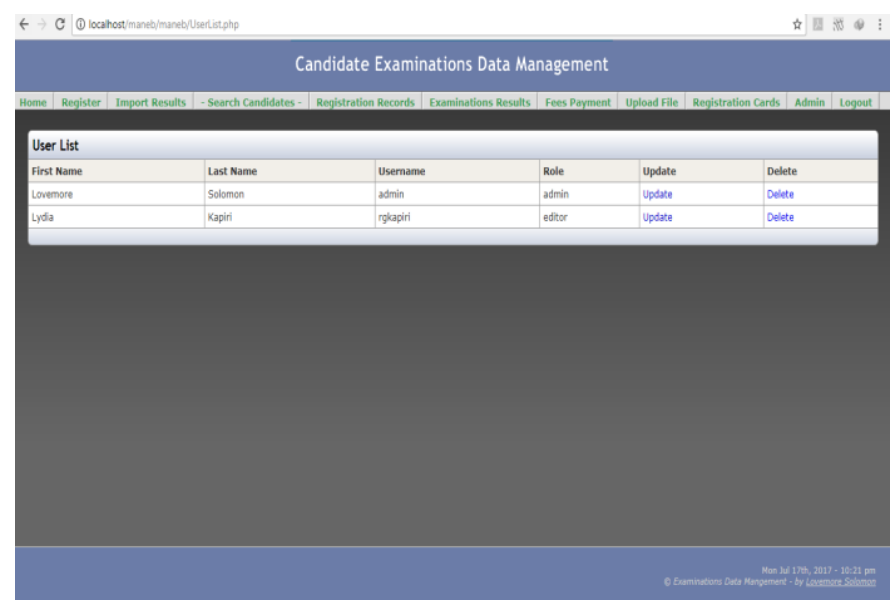

Fig. 20. Viewing users of the system.

The Web application is also used to view individual or all registered candidates as well as examinations results as illustrated in Fig. 21 and 22.

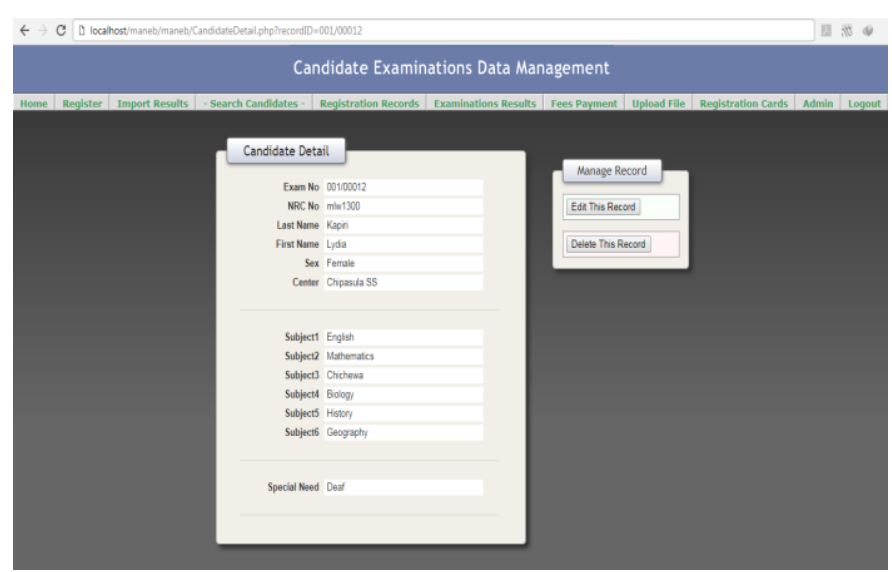

Fig. 21. Viewing individual registration record.

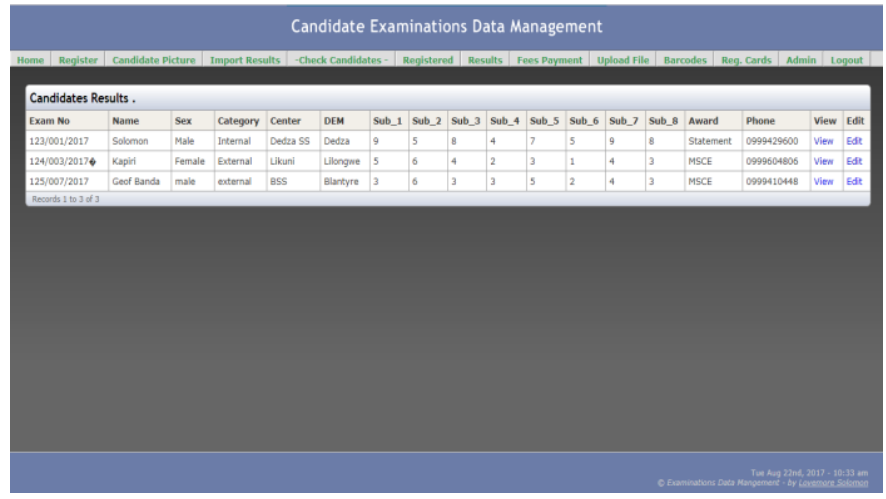

Fig. 22. Viewing candidates' examinations results.

When marking and grading of examinations scripts are completed, candidates' results are imported into the system (Fig. 23) so that they can be sent via SMS/USSD. 


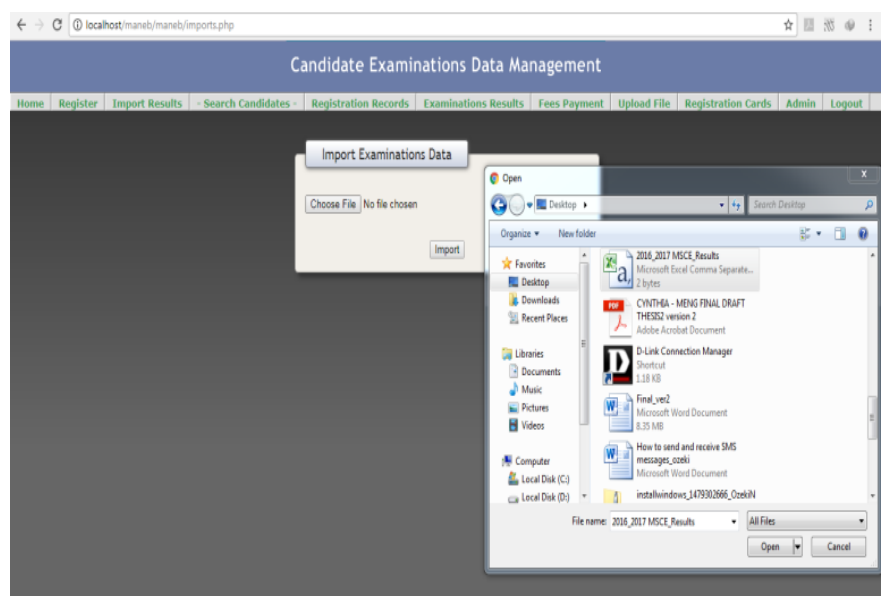

Fig. 23. Importing examinations results.

After registration, candidates' registration cards will be generated from the system. The cards will have barcodes with examination numbers imbedded. The cards will be used to validate candidates on the day of writing examinations by scanning the barcodes. Fig. 24 is an example registration card generated from the system.

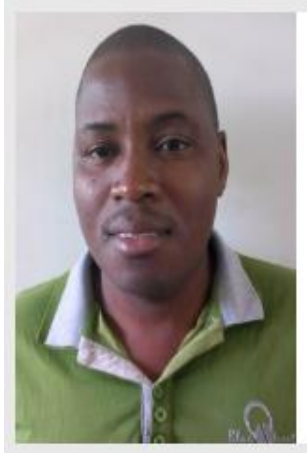

Fig. 24.

\section{MANEB Student Identity Card \\ Name: Lovemore Solomon \\ Birth Date:08/07/2017 \\ Sex: $\quad$ Male \\ Category: Internal \\ Center: Dedza SS \\ DEM: Dedza}

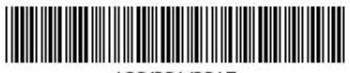

$123 / 001 / 2017$

Candidate registration card.

The system prototype was tested using dummy data. During the testing, fifty records were registered. The system was deployed on a server and accessed remotely to simulate the cloud environment. It was shown that using mobile SMS or USSD tremendously reduces the time it takes to register candidates. The system prototype's performance was measured in terms of its throughput, response time and error rate which were compared with those of the current system.

\section{DISCUSSION AND CONCLUSION}

\section{A. Discussion}

The study was conducted to establish the challenges faced by MANEB regarding registration, verification of registration details as well as dissemination of examinations results. The challenges were established through a base line study that was undertaken in some selected districts. Literature reviewed during the study unleashed that similar challenges are also encountered in other countries particularly in Africa. The rating of the current system was based on the effectiveness of the registration procedures, verification of registration details and dissemination of examinations results. The study also revealed that in many schools, some candidates have failed to sit the national examinations as a result of irregularities in the current system. During the study, the business process of MANEB based on the current system was developed and this assisted the researcher to develop a model of the proposed system. Almost all the respondents during the study indicated that they possess and know how to use a mobile phone. About $95 \%$ of the respondents recommended the introduction of the proposed system in order to curb the challenges currently experienced. From the results got, it is clearly seen that a Web based system integrated with SMS or USSD can provide a more convenient and robust method of registering students as well as disseminating examinations results. This fully automated system can help in the reduction of mistakes that occur as a result of manual processes currently being used.

\section{B. Conclusion}

In an effort to enhance the administration of national examinations more especially management of candidates' registration and examinations results data, MANEB should strive to use modern technologies such as mobile applications and cloud computing. In the study, a baseline survey was conducted to establish the challenges faced by MANEB regarding registration, verification of registration details as well as dissemination of examinations results. The baseline study, literature reviewed and the system prototype development enabled the researcher to meet the objectives of the study. The objectives include establishing the challenges currently faced by MANEB in managing and disseminating candidates' data, designing a model based on cloud architecture and SMS/USSD mobile application to address the challenges, mapping the current business process for MANEB using the model developed and finally building a prototype based on the business process and the model. The prototype developed was tested and proved to be more efficient than the current system.

\section{RECOMMENDATIONS AND FUTURE WORKS}

The researcher made the following recommendations after carrying out the study.

a) MANEB, through the Ministry of Education, Science and Technology should lobby for more funding from Government to implements the system.

b) The Government through the Malawi Communications Regulatory Authority (MACRA) should urge telecommunications companies in the country to extend their network coverage to the remotest areas so that wherever there are settlements, people should have mobile network in order to be able to use the system.

c) The Government of Malawi should expedite the egovernment project which will be implemented using cloud environment so that the system should be deployed in the same environment.

Since the proposed system only looks at the registration, verification and dissemination of results, future works should also focus on having a single and complete examinations management system that will also automate the processes like online taking of examinations by candidates as well as grading and scoring of examinations scripts. From security point of view, future works should consider incorporation of GPS/GIS 
to be able to track and record the position a candidate is registering from as well as the use of biometrics to capture the physical part of registering candidates.

\section{ACKNOWLEDGEMENT}

The researcher was sponsored by the Government of Malawi through the Department of Human Resources Management and Training to do his studies with the University of Zambia.

\section{REFERENCES}

[1] V. M. Chalila and M. Nkhoma, "Ensuring effectiveness of assessment and certification in achieving educational, social and economic goals: The case of the Malawi National Examinations Board," in 21st Conference of the Association of Educational Assessment in Africa (AEAA), Cape Town, 2003.

[2] R. Sabates, "School Dropout in Bangladesh: Insights using panel data," International Journal of Educational Development, vol. 33, pp. 225-232, 2013.

[3] C. Kirazoglu, "The investigation of school-dropout at the secondary level of formal education: The stated reasons by school administrators and school counselors: A preliminary study," Procedia Social and Behavioral Sciences, vol. 1, pp. 905-914, 2009.

[4] P. Mell and T. Grance, "The NIST Definition of Cloud Computing," National Institute of Standards and Technology, Gaithersburg, 2011.

[5] World Bank, "Malawi National Education Profile 2014 Update," FHI Education Center and Data Policy, Lilongwe, 2014.

[6] W. Witte, "A critical review of literature on dropout," Educational Research Review, vol. 10, pp. 13-28, 2013.

[7] K. Taniguchi, "Determinants of repetition in primary school in subSaharan Africa: An event history analysis for rural Malawi," International Journal of Educational Development, vol. 45, pp. 98-111, 2015.

[8] R. Sabates, "School Dropout: Patterns, Causes, Changes and Policies," Center for International Education, 2010.

[9] R. S. Maposa and F. Sibanda, "The Ethics of ICT Assessment in Public Examinations: Reflections on the Zimbabwean Experience," International Journal of Academic Research in Progressive Education and Development, vol. 2, no. 1, 2013.

[10] I. O. Iluobe, "ICT as a panacea to examination malpractice," in 30th conference of the Association for Educational Assessment in Africa (AEAA), Gaborone, 2012.

[11] P. Mell and T. Grance, "The NIST Definition of Cloud Computing," National Institute of Standards and Technology, Gaithersburg, 2011.

[12] K. S. Rao and R. K. Challa, "Adoption of Cloud Computing in Education and Learning," International Journal of Advanced Research in Computer and Communication Engineering, vol. 2, no. 10, 2013.

[13] J. Strickland, "How Cloud computing works," January 2010.[Online].Available: http://computer.howstuffworks.com/cloudcomputing.htm. [Accessed 17 December 2016].

[14] M. Armbrust, "Above the Clouds: A Berkeley View of Cloud Computing," Electrical Engineering and Computer Sciences, University of California, Berkeley, 2009.
[15] Z. Zafar et al, "Cloud Computing Services for the Healthcare Industry," International Journal of multidisciplinary sciences and engineering, vol. 5, no. 7, 2014.

[16] K. S. Rao and R. K. Challa, "Adoption of Cloud Computing in Education and Learning," International Journal of Advanced Research in Computer and Communication Engineering, vol. 2, no. 10, 2013.

[17] S. A. Madani et al, "A survey of Mobile Cloud Computing Application Models," IEEE Communications Survey and Tutorials, vol. 16, no. 1, 2014.

[18] Y. Al-Bastaki and A. Al-Ajeeli, "A framework for WAP-based course registration system," Elsevier Computers and Education, vol. 44, pp. 327-342, 2005.

[19] M. A. Shareef, "Reformation of public service to meet citizens' needs as customers: Evaluating SMS as an alternative service delivery channel," Elsevier - Computers in Human Behavior, vol. 61, pp. 255-270, 2016.

[20] A. Acker, "The Short Message Service: Standards, infrastructure and innovation," Elsevier - Telematics and Informatics, vol. 31, pp. 559-568, 2014.

[21] S. F. Persada et al, "Toward Paperless Public Announcement on Environmental Impact Assessment (EIA) through SMS Gateway in Indonesia," Procedia - Environmental Sciences, vol. 20, pp. 271-279, 2014.

[22] J. Brown, B. Shipman and R. Vetter, "SMS: The Short Message Service," University of North Carolina, Wilmington.

[23] O. Awodele, "An Improved SMS User Interface Result Checking System," Interdisciplinary Journal of Information, Knowledge and Management, vol. 4, 2009.

[24] H. Rongyu et al, "A PK-SIM card based end-to-end security framework for SMS," Computer Standards and Interfaces, vol. 31, pp. 629-641, 2009.

[25] C. L. Tseng, "Feasibility study on application of GSM-SMS technology to field data acquisition," Computers and electronics in agriculture, vol. 53, pp. 45-59, 2006.

[26] L. Boukas, "Pandora: An SMS-oriented m-information system for educational realms," Journal of Network and Computer Applications, vol. 32, pp. 684-702, 2009.

[27] M. Shamsuzzaman et al, "Use of mobile phones for improving vaccination coverage among children living in rural hard-to-reach areas and urban streets of Bangladesh," Elsevier - Vaccine, vol. 34, pp. 276283, 2016.

[28] Prajakta et al, "Online Healthcare System Using the Concept of Cloud Computing," International Journal of Innovative Research in Electrical, Electronics, Instrumentation and Control Engineering, vol. 3, no. 10, 2015.

[29] E.Taskin, "GSM MSC/VLR Unstructured Supplementary Service Data (USSD) Service," Uppsala Universitet, Uppsala.

[30] A. Dabas and C. Dabas, "Implementation of Real Time Tracking using Unstructured Supplementary Service Data," World Academy of Science, Engineering and Technology, vol. 54, 2009.

[31] Z. Wang and H. Gu, "A Wireless Medical Information Query system based on Unstructured Supplementary Service Data (USSD)," Dulian University of Technology, Lianing . 